\title{
New measurements of cosmic ray air showers with the digital radio interferometer LOPES
}

\author{
F. G. Schröder ${ }^{1}$, W. D. Apel ${ }^{1}$, J. C. Arteaga ${ }^{2, a}$, T. Asch $^{3}$, L. Bähren ${ }^{4}$, K. Bekk ${ }^{1}$, M. Bertaina ${ }^{5}$, P. L. Biermann ${ }^{6}$, \\ J. Blümer ${ }^{1,2}$, H. Bozdog ${ }^{1}$, I. M. Brancus ${ }^{7}$, P. Buchholz ${ }^{8}$, S. Buitink ${ }^{4}$, E. Cantoni ${ }^{5,9}$, A. Chiavassa ${ }^{5}$, K. Daumiller ${ }^{1}$, \\ V. de Souza ${ }^{2, b}$, P. Doll ${ }^{1}$, R. Engel ${ }^{1}$, H. Falcke ${ }^{4,10}$, M. Finger ${ }^{1}$, D. Fuhrmann ${ }^{11}$, H. Gemmeke ${ }^{3}$, C. Grupen ${ }^{8}$, A. Haungs ${ }^{1}$, \\ D. Heck ${ }^{1}$, J. R. Hörandel ${ }^{4}$, A. Horneffer ${ }^{4}$, D. Huber ${ }^{2}$, T. Huege ${ }^{1}$, P. G. Isar ${ }^{1, \mathrm{c}}$, K.-H. Kampert ${ }^{11}$, D. Kang ${ }^{2}$, \\ O. Krömer ${ }^{3}$, J. Kuijpers ${ }^{4}$, S. Lafebre ${ }^{4}$, K. Link ${ }^{2}$, P. Luczak ${ }^{12}$, M. Ludwig ${ }^{2}$, H.J. Mathes ${ }^{1}$, M. Melissas ${ }^{2}$, C. Morello ${ }^{9}$, \\ S. Nehls ${ }^{1}$, J. Oehlschläger ${ }^{1}$, N. Palmieri ${ }^{2}$, T. Pierog ${ }^{1}$, J. Rautenberg ${ }^{11}$, H. Rebel ${ }^{1}$, M. Roth ${ }^{1}$, C. Rühle ${ }^{3}$, A. Saftoiu ${ }^{7}$, \\ H. Schieler ${ }^{1}$, A. Schmidt ${ }^{3}$, O. Sima ${ }^{13}$, G. Toma ${ }^{7}$, G. C. Trinchero ${ }^{9}$, A. Weindl ${ }^{1}$, J. Wochele $^{1}$, M. Wommer ${ }^{1}$, \\ J. Zabierowski ${ }^{12}$, and J. A. Zensus ${ }^{6}$ \\ ${ }^{1}$ Karlsruhe Institute of Technology (KIT) - Campus North, Institut für Kernphysik, Germany \\ ${ }^{2}$ Karlsruhe Institute of Technology (KIT) - Campus South, Institut für Experimentelle Kernphysik, Germany \\ ${ }^{3}$ Karlsruhe Institute of Technology (KIT) - Campus North, Institut für Prozessdatenverarbeitung und Elektronik, Germany \\ ${ }^{4}$ Radboud University Nijmegen, Department of Astrophysics, The Netherlands \\ ${ }^{5}$ Dipartimento di Fisica Generale dell' Universita Torino, Italy \\ ${ }^{6}$ Max-Planck-Institut für Radioastronomie Bonn, Germany \\ ${ }^{7}$ National Institute of Physics and Nuclear Engineering, Bucharest, Romania \\ ${ }^{8}$ Universität Siegen, Fachbereich Physik, Germany \\ ${ }^{9}$ INAF Torino, Istituto di Fisica dello Spazio Interplanetario, Italy \\ ${ }^{10}$ ASTRON, Dwingeloo, The Netherlands \\ ${ }^{11}$ Universität Wuppertal, Fachbereich Physik, Germany \\ ${ }^{12}$ Soltan Institute for Nuclear Studies, Łódź, Poland \\ ${ }^{13}$ University of Bucharest, Department of Physics, Bucharest, Romania \\ ${ }^{a}$ now at: Universidad Michoacana, Instituto de Física y Matemáticas, Mexico \\ ${ }^{b}$ now at: Universidade São Paulo, Instituto de Física de São Carlos, Brasil \\ ${ }^{c}$ now at: Institute of Space Science, Bucharest, Romania
}

Received: 18 October 2010 - Revised: 3 May 2011 - Accepted: 10 May 2011 - Published: 31 August 2011

\begin{abstract}
LOPES is a digital radio interferometer which measures the radio emission of extensive cosmic ray air showers. It mainly consists of 30 dipole antennas installed in co-location with KASCADE-Grande at the Karlsruhe Institute of Technology (KIT) in Germany. KASCADE-Grande measures the secondary air shower particles at ground. Whenever KASCADE-Grande detects a high-energy cosmic ray event $\left(\gtrsim 10^{16} \mathrm{eV}\right)$, it triggers LOPES which then digitally records the radio signal in the frequency band from 40 to $80 \mathrm{MHz}$. Using interferometric methods, LOPES is able to successfully detect air shower induced radio pulses, even in the noisy environment at the KIT. In the present studies, a considerable progress in understanding the radio emission mechanism is shown: The latest version of the "radio emis-
\end{abstract}

Correspondence to: F G. Schröder (frank.schroeder@kit.edu) sion in air shower" simulation program, REAS3, seems to be the first Monte Carlo tool which is able to reproduce the magnitude and slope of most of the measured lateral distributions.

\section{Introduction}

Radio emission of extensive air showers (EAS) initiated by high-energy cosmic rays has been discovered more than forty years, ago - for an overview see Melissas et al. (this issue). LOPES is a modern, digital radio interferometer for air shower measurements. It was built in 2003 at the location of the well established EAS experiment KASCADE-Grande (Apel et al., 2010a). LOPES started by using prototype hardware of LOFAR, a digital radio observatory with cosmic ray detection among its key science projects (Falcke et al., 2007). Recent results from CODALEMA, another successful digital radio array for EAS detection, can be found in Ravel et al.

Published by Copernicus Publications on behalf of the Arbeitsgemeinschaft Extraterrestrische Forschung e.V. 


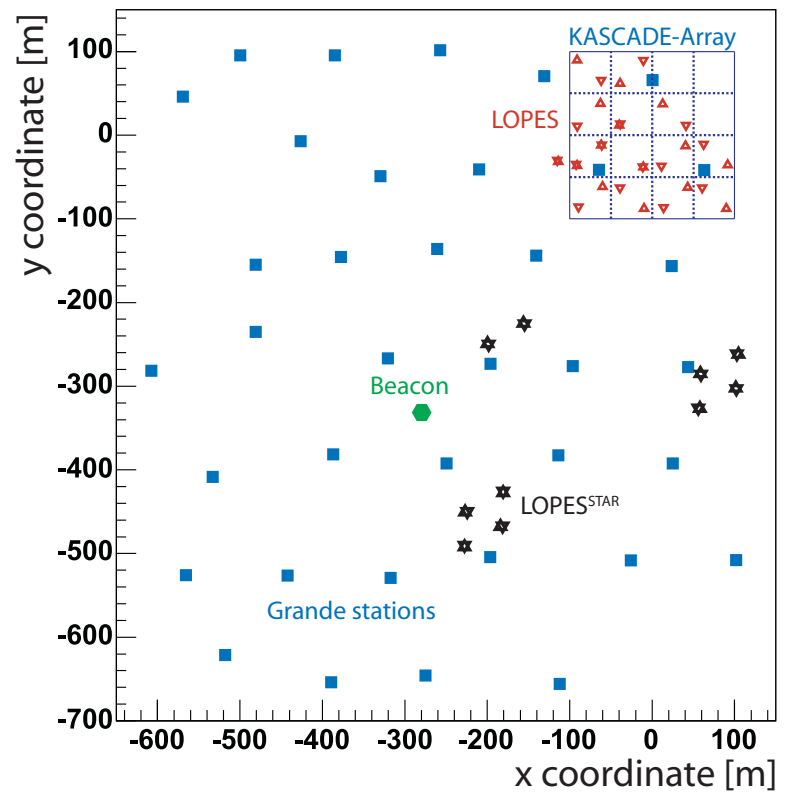

Fig. 1. Layout of LOPES until end of 2009: LOPES antennas are positioned within the KASCADE-Grande experiment at the Karlsruhe Institute of Technology. Stars mark two antennas with eastwest and north-south alignment at the same position, triangles antennas with either one alignment.

(2010). ANITA, a balloon borne radio experiment above the Antarctica, has recently claimed seeing high-energy cosmic rays by the reflection of the EAS radio signal at the ice surface (Hoover et al., 2010). The radio technique is meanwhile seen as a new path in the detection of highest energy cosmic particles, as well as in the observation of radio signals generated by cosmic rays and neutrinos impacting the lunar regolith - see e.g. Mevius et al. (2010).

LOPES successfully proved first that digital radio interferometry is indeed feasible for EAS detection (Falcke et al., 2005). After this proof-of-principle, LOPES focused mainly on three issues: investigating how the direction, energy and mass of the primary particle can be reconstructed, understanding the radio emission mechanism in detail, and developing hardware and techniques for the next generation radio arrays like LOFAR and the Auger Engineering Radio Array (AERA) (Fliescher et al., 2010). A recent overview of LOPES results can be found in Huege et al. (2010a). The present article focuses on an update on the improved analysis of radio lateral distributions measured with LOPES and a comparison of these to Monte Carlo radio simulations performed with REAS3 (Ludwig and Huege, 2010).

\section{Setup and preparatory work}

After an initial phase of 10 operating antennas from 2003 to 2005, LOPES was upgraded to 30 absolute amplitude cali- brated dipole antennas (Fig. 1) (Nehls et al., 2007), and by a new type of antenna forming the LOPES ${ }^{\text {STAR }}$ setup (Rühle et al., 2010) - dedicated to technical developments like selftriggering. For the main part of LOPES inverted v-shaped dipole antennas were used until the end of 2009. At the beginning, all antennas have been aligned in the east-west direction because the geomagnetic radio emission of EAS is predominantly east-west polarized, see Huege et al. (2010b) for a recent review of EAS radio emission models. At the end of 2006, half of the antennas have been rotated by $90^{\circ}$ to measure also the north-south polarization component, and in 2010, the antenna type has been changed to a tripole antenna which consists of three crossed dipoles, and which can measure the complete polarization of the electric field vector. An important goal of the polarization measurements is a better understanding of the radio emission process. A detailed analysis of the polarization measurements is still in progress. Thus, in this article we will present results obtained with east-west aligned antennas, only.

For all setups, LOPES is triggered by KASCADE-Grande, and digitally records the radio signal in the frequency band of $40-80 \mathrm{MHz}$ with a sampling rate of $80 \mathrm{Megasamples}$ per second, i.e. it operates in the second Nyquist domain, and the radio field strength in-between the sampled data points can be retrieved by up-sampling algorithms. One of the key features of LOPES is the possibility to combine the information of all antennas in a subsequent analysis by calculating a cross-correlation beam, which is formed into the shower direction known from KASCADE-Grande and optimized by a fit. This makes LOPES a digital radio interferometer, and it is essential to improve the signal-to-noise ratio in the noisy environment of Karlsruhe. It is only possible because of a remarkable relative timing precision of $\sim 1 \mathrm{~ns}$ which is achieved with a reference beacon continuously emitting sine waves (Schröder et al., 2010a). Measuring the phasing of these sine waves in each antenna allows to permanently monitor the relative timing precision and to improve it to the desired level.

LOPES-10 has already established the proof-of-principle that cosmic ray radio detection is possible with a digital radio interferometer (Falcke et al., 2005). It could confirm results of earlier experiments that the radio emission is predominantly of geomagnetic origin, since its amplitude depends on the geomagnetic angle (= angle between shower axis and the Earth's magnetic field). Furthermore, LOPES has shown that the radio amplitude is roughly proportional to the primary energy - as expected for any coherent radio emission process - and that the arrival direction can be reconstructed with an accuracy of $\lesssim 1.5^{\circ}$ (Nigl et al., 2008). For the reconstruction of the primary mass there are two promising ways under study: First the shape of the radio wavefront (Lafebre et al., 2010; Schröder et al., 2010c), and second the slope of the lateral distribution of the radio emission (Huege et al., 2008). The evaluation of the first way is still in progress, but results from the latter one are presented in the next paragraph. 


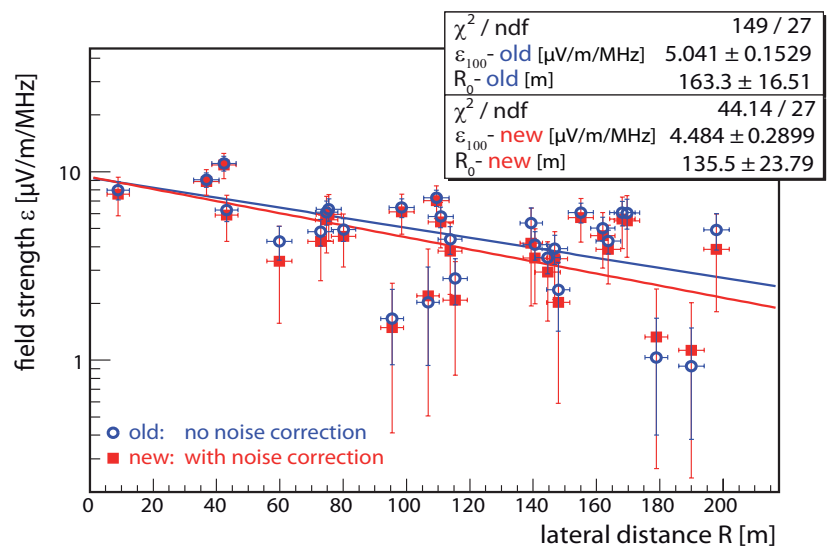

Fig. 2. Example lateral distribution with and without correction of the noise influence. Noise usually increases the amplitude except for very low signal-to-noise ratios $(<1)$ for which a decrease due to destructive interference is more likely.

\section{Lateral distribution of the radio signal}

The lateral distribution can be determined for high-energy events with $E \gtrsim 10^{17} \mathrm{eV}$, for which the signal-to-noise ratio is sufficient to measure the amplitude of the radio pulse at individual antennas (Apel et al., 2010b). By fitting an exponential lateral distribution function (LDF), it was confirmed that the amplitude of most events decays approximately exponentially - with the exception of $\lesssim 20 \%$ of the events for which the observed lateral distribution is either flat or flattens towards the shower core. Thus, an exponential LDF is only a simplified but for most events sufficient approximation to the real LDFs.

Because of the noisy environment of LOPES, special care has to be paid on the treatment of noise, when reconstructing the lateral distribution. As noise can interfere constructively or destructively with the measured signal, a coherent approach for determining the noise level, for a correction procedure, and the estimation of the correction uncertainties were developed (Schröder et al., 2010b,c). Noise is not only the dominant source for uncertainties in pulse time and amplitude measurements, but it also systematically flattens the lateral distributions by typically $\sim 10 \%$. In Fig. 2 an example is shown where the new noise treatment is compared to the old one for the same event. The error bars on a single measurement (individual antenna) is now larger in most cases, but as now the noise influence on the amplitude is really corrected also the value is different. Where the first naturally decreases the $\chi^{2}$ of the LDF fit, the latter is changing the slope of the lateral distribution, which is the most sensitive parameter for mass sensitivity of the detection technique, and therefore of great importance for the analysis.

After correcting the measured lateral distributions for noise and fitting an exponential function, they are compared with detailed REAS3 Monte Carlo simulations (Lud-

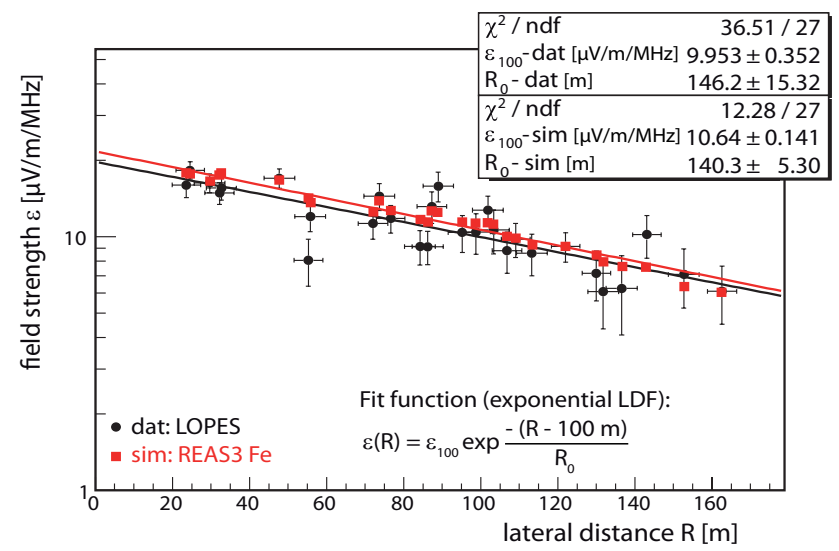

Fig. 3. Comparison of the lateral distribution of an individual event measured with LOPES and simulated with REAS3 for an iron nucleus as primary particle.

wig and Huege, 2010). As input for the simulations of the individual showers the arrival direction, point of incidence with the Earth's surface, and primary energy given by the KASCADE-Grande reconstruction are used. As primary mass, both protons (p) and iron nuclei $(\mathrm{Fe})$ as most abundant cosmic rays in this energy range are chosen. Such a detailed comparison was performed for a selection of 81 events with a high signal-to-noise ratio (Figs. 3 and 4). Most of them agree very well with the REAS3 simulations showing that the simulations well reproduce the order of magnitude of the amplitude parameter $\epsilon_{100}$ and the slope parameter $R_{0}$ of the exponential LDF fit - with the exception that REAS3 can reproduce relatively flat lateral distributions ( $\widehat{=}$ large $R_{0}$ ) only in some cases. Furthermore, the slope parameter shows a mass sensitivity: proton showers show a slightly steeper lateral distribution, which confirms that a measurement of the lateral distribution is one possible way to estimate the primary mass. However, the present uncertainties do not allow a definite quantitative conclusion on the primary composition of the selected LOPES events.

\section{Conclusions}

LOPES has shown that EAS can be measured with digital, interferometric radio antenna arrays, and that it is possible to reconstruct the energy and arrival direction of the primary cosmic ray particles. In addition to theoretical predictions, there are first experimental hints that also the primary mass can be reconstructed with radio measurements, which would make radio measurements a complementary detection technique for cosmic rays, among particle surface detectors, fluorescence and Cherenkov telescopes. It could be shown that the primary mass can in principle be estimated by measuring the slope of the lateral distributions of the radio signal. However, the achievable precision is still under investigation. 

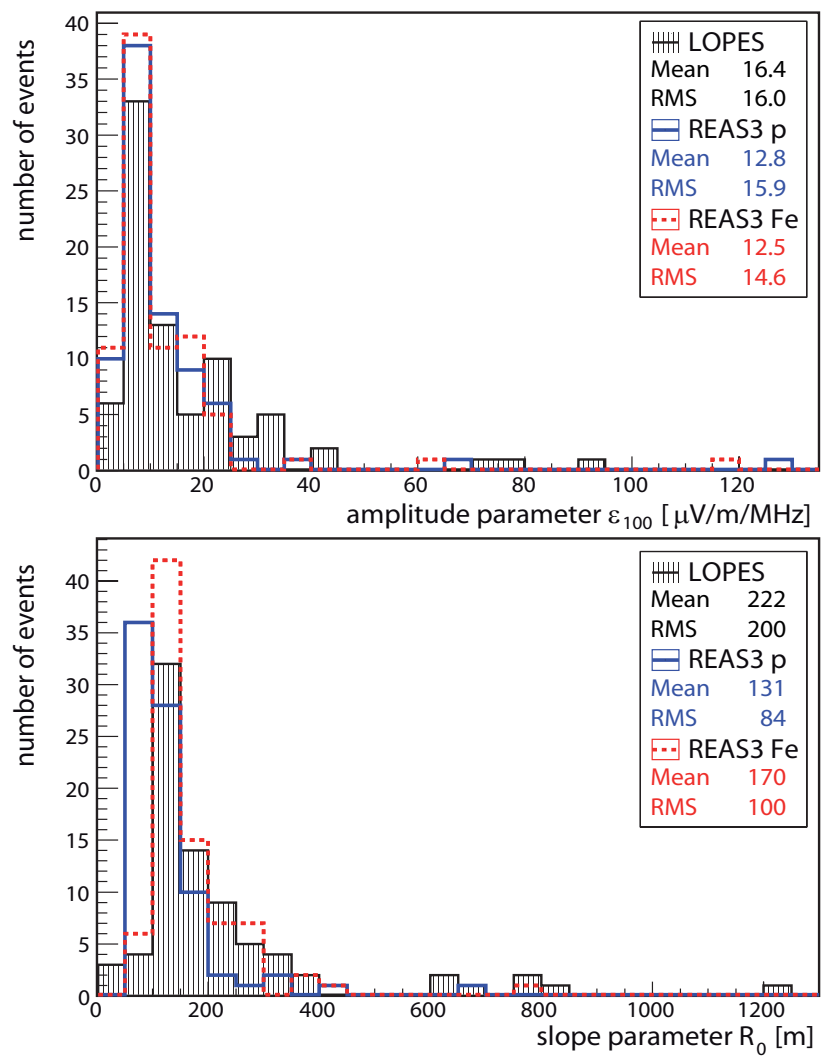

Fig. 4. LDF amplitude and slope parameter distributions for measured and simulated radio detected air showers.

For competing with the well established detection methods, radio measurements of EAS have to be not only possible, but also precise enough, which requires accurate measurements as well as a sufficient understanding of the radio emission mechanisms. In both domains, LOPES has made remarkable progress. Newly developed methods for amplitude and time calibration (Nehls et al., 2007; Schröder et al., 2010a), as well as for noise treatment (Schröder et al., 2010b) have greatly decreased systematical uncertainties of the radio measurements. This allows testing recently improved emission models with an adequate accuracy. This way, an up to now unachieved agreement between data and new REAS simulations could be observed: REAS3 generally is able to reproduce radio amplitudes measured with LOPES within the experimental uncertainties.

Edited by: T. Laitinen

Reviewed by: three anonymous referees

\section{References}

Apel, W. D., et al. (KASCADE-Grande Coll.): The KASCADEGrande experiment, Nucl. Instr. Meth. A, 620, 202-216, 2010a.

Apel, W. D., et al. (LOPES Coll.): Lateral distribution of the radio signal in extensive air showers measured with LOPES, Astropart. Phys., 32, 294-303, 2010b.
Falcke, H., et al. (LOPES Coll.): Detection and imaging of atmospheric radio flashes from cosmic ray air showers, Nature, 435, 313-316, 2005.

Falcke, H., van Haarlem, M. P., de Bruyn, A. G., et al.: A very brief description of LOFAR - the Low Frequency Array, Highlights of Astron., 14, 386-387, 2007.

Fliescher, S., et al. (Pierre Auger Coll.): Radio Detection of Cosmic-Ray-Induced Air Showers at the Pierre Auger Observatory, Nucl. Instr. Meth. A, doi:10.1016/j.nima.2010.11.045, 2010.

Hoover, S., et al. (ANITA Coll.): Observation of Ultrahigh-Energy Cosmic Rays with the ANITA Balloon-Borne Radio Interferometer, Phys. Rev. Lett., 105, 151101, 2010.

Horneffer, A., et al. (LOPES Coll.): Primary Particle Energy Calibration of the EAS Radio Pulse Height, in: Proc. 30th ICRC, 4, 83-86, 2007.

Huege, T., Ulrich, R., Engel, R.: Dependence of geosynchrotron radio emission on the energy and depth of maximum of cosmic ray showers, Astropart. Phys., 30, 96-104, 2008.

Huege, T., et al. (LOPES Coll.): The LOPES experiment - recent results, status and perspectives, Nucl. Instr. Meth. A - ARENA proc. in press, doi:10.1016/j.nima.2010.11.081, 2010a.

Huege, T., Ludwig, M., Scholten, O., de Vries, K. D.: The convergence of EAS radio emission models and a detailed comparison of REAS3 and MGMR simulations, Nucl. Instr. Meth. A ARENA proc. in press, doi:10.1016/j.nima.2010.11.041, $2010 \mathrm{~b}$.

Lafebre, S., Falcke, H., Hörandel, J. R., et al.: Prospects for determining air shower characteristics through geosynchrotron emission arrival times, Astropart. Phys., 34, 12-17, 2010.

Ludwig, M. and Huege, T.: REAS3: Monte Carlo simulations of radio emission from cosmic ray air showers using an 'endpoint' formalism, Astropart. Phys., 34, 438-446, 2011.

Melissas, M., et al. (Pierre Auger Coll.): Overview of radiodetection of cosmic ray air showers and prospects for a larger scale experiment, ASTRA, 7, 207-210, 2011.

Mevius, M., Buitink, S., Falcke, H., et al.: Detecting ultra high energy neutrinos with LOFAR, Nucl. Instr. Meth. A - ARENA proceedings in press, doi:10.1016/j.nima.2010.11.018, 2010.

Nehls, S., Hakenjos, A., Arts, M.J., et al.: Amplitude calibration of a digital radio antenna array for measuring cosmic ray air showers, Nucl. Instr. Meth. A, 589, 350-361, 2008.

Nigl, A., et al. (LOPES Coll.): Direction identification in radio images of cosmic-ray air showers detected with LOPES and KASCADE, Astron. Astroph., 487, 781-788, 2008.

Ravel, O., et al. (CODALEMA Coll.): The CODALEMA experiment, Nucl. Instr. Meth. A - ARENA proceedings in press, doi:10.1016/j.nima.2010.12.057, 2010.

Rühle, C., et al. (Pierre Auger Coll.): Advanced digital selftriggering of radio emission of cosmic rays, Nucl. Instr. Meth. A - ARENA proc. in press, doi:10.1016/j.nima.2010.11.017, 2010.

Schröder, F. G., Asch, T., Bähren, L., et al., New method for the time calibration of an interferometric radio antenna array, Nucl. Instr. Meth. A, 615, 277-284, 2010a.

Schröder, F. G., et al. (LOPES Coll.): On noise treatment in radio measurements of cosmic ray air showers, Nucl. Instr. Meth. A ARENA proc. in press, doi:10.1016/j.nima.2010.11.009, 2010b.

Schröder, F. G., Instruments and Methods for the Radio Detection of High Energy Cosmic Rays, Ph.D. thesis, Karlruhe Institute of Technology (KIT), Germany, available at http://www.astro.ru.nl/ lopes/publications/phd_theses, 2010c. 